\title{
Validación del M-CHAT-R/F como instrumento de tamizaje para detección precoz en niños con trastorno del espectro autista
}

\author{
M-CHAT-R/F Validation as a screening tool for early detection in children \\ with autism spectrum disorder
}

\author{
María Elisa Coelho-Medeiros ${ }^{\mathrm{a}}$, Jonathan Bronstein ${ }^{\mathrm{a}}$, Karina Aedo ${ }^{\mathrm{a}}$, Jaime A. Pereira ${ }^{\mathrm{a}, \mathrm{g}}$, Verónica Arraño ${ }^{\mathrm{b}}$, \\ Carolina A. Perez ${ }^{\mathrm{c}}$, Patricia M. Valenzuela ${ }^{\mathrm{d}}$, Rosario Moore ${ }^{\mathrm{d}}$, Isabel Garrido ${ }^{\mathrm{e}}$, Paula Bedregal ${ }^{\mathrm{f}}$
}

\begin{abstract}
aDepartamento de Psiquiatría, Unidad de Psiquiatría del Niño y del Adolescente. Escuela de Medicina, Pontificia Universidad Católica de Chile bTerapeuta Ocupacional. Departamento de Psiquiatría, Escuela de Medicina, Pontificia Universidad Católica de Chile Interna de Medicina. Facultad de Medicina, Pontificia Universidad Católica de Chile dDepartamento de Pediatría, Escuela de Medicina, Pontificia Universidad Católica de Chile eEnfermera Universitaria. Departamento de Psiquiatría, Escuela de Medicina, Pontificia Universidad Católica de Chile fDepartamento de Salud Pública, Escuela de Medicina, Pontificia Universidad Católica de Chile ILaboratorio de Interfaces Cerebro Computador y Neuromodulación y Centro Interdisciplinario de Neurociencias, Escuela de Medicina, Pontificia Universidad Católica de Chile.
\end{abstract}

Recibido el 27 de abril de 2018; aceptado el 23 de octubre de 2018

\section{Resumen}

Introducción: El tamizaje de los Trastornos del Espectro Autista (TEA) mediante el Modified Checklist for Autism in Toddlers - Revised with Follow Up (M-CHAT-R/F) aumenta la detección precoz, posibilitando intervenciones tempranas y mejorando el pronóstico. Este instrumento es parte del algoritmo de manejo ante la sospecha de TEA en diversas guías clínicas. El objetivo fue realizar la validación concurrente, discriminante y el análisis de confiabilidad del M-CHAT-R/F en una población chilena. Pacientes y Método: Esta es la segunda etapa de la adaptación transcultural, de diseño transversal. Se aplicó M-CHAT-R/F a una muestra de 20 niños con sospecha de TEA y 100 niños de control sano seleccionados al azar, de 16-30 meses de edad. Se aplicó Autism Diagnostic Observation Schedule (ADOS-2), considerado como referencia, a los 20 pacientes de la muestra clínica, a 20 niños de la muestra de control sano y a aquellos casos de la muestra de control sano con M-CHAT-R/F positivo. Se calculó alfa de Cronbach, análisis de correlación de M-CHAT-R/F y ADOS-2 y sensibilidad y especificidad. Resultados: En el grupo de control sano, M-CHAT-R/F resultó alterado en 2 pacientes, siendo uno positivo y otro negativo para TEA con ADOS-2. En muestra clínica el M-CHAT-R/F fue positivo en todos, con test de ADOS-2 negativo en 3 casos. La confiabilidad Alfa del M-CHAT$\mathrm{R} / \mathrm{F}$ fue $=0,889$, la sensibilidad y especificidad discriminante de 100 y $98 \%$ y la concurrente $100 \%$ y $87,5 \%$ respectivamente. Conclusión: M-CHAT-R/F en su versión chilena resultó fiable, sensible y específico de manera similar al original, lo cual abre la posibilidad de su utilización en población clínica y para investigación. La validación es un proceso continuo que se debe profundizar.
Palabras clave:

Autismo;

Trastorno del Espectro

Autista;

Tamizaje;

Psiquiatría Infantil;

Desarrollo Infantil 


\begin{abstract}
Introduction: Screening for Autism Spectrum Disorders (ASD) using the Modified Checklist for Autism in Toddlers, Revised with Follow-up (M-CHAT-R/F) increases early detection, allowing early interventions and improving prognosis. This tool is part of the management in case of suspected ASD in several clinical guidelines. The objective of this article was to conduct the concurrent and discriminant validation and the reliability analysis of M-CHAT-R/F in the Chilean population. Patients and Method: This is the second stage of the cross-cultural adaptation of cross-sectional design. M-CHAT$\mathrm{R} / \mathrm{F}$ was applied to a sample of 20 children with suspected ASD and 100 randomly selected healthy control children, aged between 16-30 months. Autism Diagnostic Observation Schedule (ADOS-2), considered as reference, was applied to the 20 patients of the clinical sample, to 20 children of the healthy control sample and to those cases of the healthy control sample with M-CHAT-R/F positive. Cronbach alpha was calculated, as well as M-CHAT-R/F and ADOS-2 correlation, sensitivity, and specificity analyses. Results: In the healthy sample, M-CHAT-R/F was positive in two patients, with one of them positive and the other one negative for ASD with ADOS-2 test. In the clinical sample, MCHAT-R/F was positive in all cases, three of them were negative in the ADOS- 2 test. The Alfa reliability of M-CHART-R/T was 0,889 , the discriminant sensitivity and specificity were $100 \%$ and $98 \%$, and the concurrent ones were $100 \%$ and $87.5 \%$ respectively. Conclusions: The Chilean M-CHAT$\mathrm{R} / \mathrm{F}$ version was reliable, sensitive and specific, similar to the original test, which opens the possibility for its use in clinical samples and for research. Validating M-CHAT-R/F is an ongoing process which must be further developed.
\end{abstract}

Keywords:

Autism;

Autism Spectrum

Disorder;

Screening;

Child Psychiatry;

Child development

\section{Introducción}

Los Trastornos del Espectro Autista (TEA) han adquirido gran relevancia como problema de salud pública debido al importante aumento en su prevalencia durante las últimas dos décadas ${ }^{1}$. Se estima que 1 cada 68 niños tiene TEA, siendo este 4 veces más frecuente en varones ${ }^{2}$. El diagnóstico de TEA no es fácil de realizar. No existen exámenes genéticos o marcadores biológicos que lo detecten y otra dificultad para su diagnóstico precoz es que durante los primeros años de vida, los niños con TEA pueden lograr un desarrollo normal en algunas áreas, sin que los padres o profesionales de salud sospechen una alteración. Los síntomas y signos sugerentes de TEA pueden ser sutiles y aparecer en forma gradual de manera que frecuentemente el diagnóstico se hace tardíamente ${ }^{3,4}$. Así, la mayoría de los niños son diagnosticados después de los 4 años de edad, cuando se hace evidente que las demandas del ambiente exceden la capacidad de respuesta del niño ${ }^{5}$.

Evidencias actuales muestran que el diagnóstico precoz de TEA mejora el pronóstico y la evolución a largo plazo de los niños que lo padecen ${ }^{6,7}$. La detección precoz permite brindar un tratamiento individualizado, multidisciplinario y oportuno que promueve un mejor desarrollo de las habilidades lingüísticas y sociales minimizando las conductas maladaptativas ${ }^{8,9}$.

La Academia Americana de Pediatría (AAP) propone para la detección precoz de TEA, realizar tamizaje universal a todos los niños a la edad de 18 y 24 meses, logrando así disminuir la brecha entre la sos- pecha, el diagnóstico y la intervención ${ }^{10,11}$. Esto refuerza la importancia de disponer de instrumentos de tamizaje confiables y adaptados a la cultura local, que puedan ser aplicados en forma universal a todos los niños en el control de salud habitual ${ }^{6,12}$. Dentro de estos instrumentos de tamizaje recomendados por la American Academy of Child and Adolescent Psychiatry (AACAP) destaca el Modified Checklist for Autism in Toddlers (M-CHAT $)^{13}$, actualmente en su versión M-CHAT-R/F (R/F: Revised with Follow-up), con sensibilidad y especificidad sobre el $80 \%$, el cual incorpora una entrevista de seguimiento (Follow-up) ${ }^{14}$. El uso de esta entrevista reduce, en gran medida, los casos de falsos positivos, evitando derivaciones innecesarias a especialistas. Además, su fácil implementación debido a una puntuación simplificada, es otra ventaja del M-CHAT-R/F respecto al M-CHAT ${ }^{14,15}$. Se aplica a niños desde los 16 y hasta los 30 meses de edad, siendo sugerido como parte del algoritmo de manejo ante la sospecha de TEA en diversas guías clínicas ${ }^{16-19}$ encontrándose validado en varios países como Argentina ${ }^{20}$, México $^{21}$, Brasil ${ }^{22}$ y España ${ }^{23}$, pero no así en Chile, a pesar de lo cual es el instrumento de tamizaje recomendado en la Guía de Práctica Clínica de Detección y Diagnóstico oportuno de los Trastornos del Espectro Autista del Ministerio de Salud de Chile (MINSAL) y en la Norma Técnica para la supervisión de niños y niñas de 0-9 años en la Atención Primaria de Salud de este país ${ }^{16,24}$.

El objetivo de este trabajo fue realizar la validación concurrente, discriminante y el análisis de confiabilidad del M-CHAT-R/F en una población chilena. 


\section{Pacientes y Método}

Se realizó un estudio en dos fases. La primera fase consistió en la adaptación cultural, desde M-CHAT$\mathrm{R} / \mathrm{F}$ versión en castellano de España. Se hizo un análisis de equivalencia de contenido, de comprensión semántica, conceptual y técnica, en una muestra intencionada de usuarios de la red UC, diseñando así el M-CHAT-R/F versión chilena ${ }^{26}$ disponible con acceso libre en la web oficial del instrumento www.mchatscreen.org ${ }^{26}$. La segunda fase, y objeto de este reporte, consistió en un estudio de diseño transversal para la validación psicométrica del instrumento M-CHAT-R y R/F. El estudio requirió de consentimiento informado del adulto responsable de cada niño participante y contó con la aprobación del comité de ética de la Pontificia Universidad Católica de Chile.

\section{Participantes}

Se realizó el tamizaje con el M-CHAT-R y R/F a una muestra no aleatoria de 120 niños entre 16 y 30 meses de edad, reclutados dentro de la Red de Salud UC Christus (Figura 1). De estos, 20 niños (muestra clínica) presentaban alta sospecha clínica de TEA luego de ser evaluados por médicos especialistas (neurólogos infantiles, psiquiatras infantiles y pediatras), según criterios del Manual Diagnóstico y Estadístico de Trastornos Mentales de la Asociación Americana de Psiquiatría en su quinta versión (DSM-5) ${ }^{27}$. Se excluyeron a pacientes con: epilepsia o tratamiento anticonvulsivante, condición médica grave o discapacidad física que imposibilitaba la aplicación de instrumento diagnóstico. La muestra de población general consistió en 100 niños que acudían a control sano o de morbilidad general, sin sospecha de TEA, excluyendo además a niños con atención en salud mental por otras causas en los 6 meses anteriores a la administración del cuestionario.

\section{Instrumentos}

- M-CHAT-R versión chilena: Cuestionario para tamizaje de TEA de 20 preguntas dicotómicas ( $\mathrm{Si}$ / No) autoaplicado por el cuidador: Puntaje $\leq 2$ es negativo. Puntaje entre 3-7 puntos significa riesgo moderado de TEA, e indica hacer entrevista de seguimiento $(\mathrm{R} / \mathrm{F})$. Puntaje $\geq 8$ puntos, se traduce en riesgo elevado para TEA e indica realizar directamente evaluación por especialista ${ }^{26}$.

- R/F versión chilena: Cuestionario estructurado de aplicación por profesional de salud tras una breve capacitación. Se preguntan sólo ítems con resultado alterado en M-CHAT-R, siguiendo el flujograma propuesto en el instrumento. Si puntaje en R/F de "NO PASA" es $\geq 2$ puntos se indica evaluación por especialista ${ }^{26}$.
- ADOS-2: La escala Autism Diagnostic Observation Schedule (ADOS) en su segunda versión ADOS2 es una evaluación estandarizada y semi estructurada de la comunicación, de la interacción social, de los intereses y del juego imaginativo, que define grado de preocupación respecto al posible diagnóstico de TEA. Es considerado el instrumento gold standard en la Guía Clínica del MINSAL ${ }^{16}$. Dado que el ADOS-2 es un instrumento semiestructurado, no existe validación específica en Chile. Ha sido establecido, dentro de las normas de su aplicación, que el evaluador realice una adecuación cultural, si es necesario, durante la sesión. La traducción al castellano del manual de este instrumento se encuentra debidamente validada ${ }^{28}$. Este instrumento de evaluación consta de un conjunto de actividades precisas, en un contexto estandarizado, donde el evaluador observa ciertas conductas relevantes para el diagnóstico TEA. Existen 4 módulos que son determinados según el nivel de lenguaje del niño y la edad. En este estudio se aplicó el módulo $\mathrm{T}$ el cual se encuentra desarrollado para menores de 30 meses, independiente de su nivel de lenguaje. Si bien, se considera a ADOS-2 como el mejor test para diagnóstico de autismo, aún el diagnóstico definitivo se basa en la evaluación clínica multidisciplinaria especializada ${ }^{29}$.

\section{Procedimiento (Figura 1)}

Se aplicó M-CHAT-R, por médicos especialistas o residentes capacitados previamente, a la totalidad de la muestra de población general y clínica. Se aplicó R/F según procedimiento del cuestionario. Se aplicó ADOS-2 a la totalidad de muestra clínica y en la muestra de población general a aquellos pacientes con MCHAT $\geq 8$ puntos o $\mathrm{R} / \mathrm{F} \geq 2$ puntos, en esos casos los padres fueron informados del riesgo que conllevaba un tamizaje positivo. Se realizó por lo tanto una evaluación, no ciega, estandarizada (ADOS-2) por tres evaluadores expertos de forma simultánea, aunque con codificación del instrumento por separado, discutiendo las discrepancias si es que las hubiere para llegar a consenso. Para el análisis discriminante, se seleccionaron al azar 20 pacientes de la muestra de la población general con M-CHAT-R negativo a quienes también se aplicó ADOS-2.

Ante la detección de otros tipos de diagnósticos clínicos durante la evaluación se realizaron las sugerencias de tratamiento y derivaciones correspondientes a cada caso particular.

\section{Análisis}

Se realizó un análisis descriptivo sociodemográfico de la muestra, y se compararon ambas submuestras utilizando pruebas no paramétricas (Prueba U de Mann- 
Whitney) y chi-cuadrado para variables nominales. Se consideró diferencias estadísticamente significativas a valores de $\mathrm{p} \leq 0,05$. Se calculó la confiabilidad interna de M-CHAT-R/F mediante alfa de Cronbach. La validez concurrente se realizó, a través de análisis de correlación, comparando los resultados de M-CHATR/F y ADOS-2 (considerado gold standard) en ambas submuestras y calculando la sensibilidad y especificidad. La validez discriminante se estimó comparando el diagnóstico clínico de alto riesgo de TEA, respecto del resultado con M-CHAT-R/F.

\section{Resultados}

\section{Características sociodemográficas}

La muestra clínica tendió a ser de niños mayores de 24 meses, en su mayoría eran hombres (95\%), los cuidadores eran de mayor edad, sin diferencias estadísticamente significativas en el nivel de estudios alcanzados y la previsión en salud (Tabla 1). Se encontraron diferencias estadísticamente significativas en la edad de la muestra general y la clínica (22 vs 24 meses respectivamente, $\mathrm{p}<0,006)$ además de diferencias en la distribución por sexo: $58 \%$ varones en la muestra general, y $95 \%$ en la muestra clínica lo que es estadísticamente significativo (chi-cuadrado, $\mathrm{p}=0,01$ ).

\section{Puntajes promedios de pruebas estudiadas}

La muestra clínica presentó puntajes promedios significativamente mayores que la muestra general, tanto de M-CHAT-R/F (9,1 vs 0,81) como en ADOS-2 (15 vs 2,7) (Tabla 2).

\section{Comportamiento de M-CHAT-R y R/F (Figura 1)}

En $90 \%$ de la muestra de la población general el M-CHAT-R fue negativo y en 10\% resultó alterado. A este grupo de pacientes según el flujograma propuesto por los autores del instrumento, se aplicó el R/F. De éstos, 7 resultaron negativos (puntaje $\leq 1$ ) y 3 pacientes siguieron presentando resultados positivos. A estos pacientes se les citó para evaluación por especialista y se les aplicó el test ADOS-2 módulo T, con el resultado en la categoría de Riesgo Severo de TEA en un caso y

Tabla 1. Características de la muestra

\begin{tabular}{|c|c|c|c|c|}
\hline & Muestra general & Muestra clínica & Total & Valor $\mathrm{p}$ \\
\hline & $\mathrm{N}: 100$ & $\mathrm{~N}: 20$ & $\mathrm{~N}: 120$ & \\
\hline Edad promedio en meses (DE) & $\begin{array}{l}22,03 \\
-4,22\end{array}$ & $\begin{array}{l}24,85 \\
-3,43\end{array}$ & $\begin{array}{l}22,47 \\
-4,22\end{array}$ & ${ }^{*} p=0,007^{1}$ \\
\hline Sexo masculino (\%) & 58,0 & 94,8 & 64,2 & ${ }^{*} p=0,001^{2}$ \\
\hline Edad promedio cuidador en años (DE) & $\begin{array}{c}28,87 \\
-13,06\end{array}$ & $\begin{array}{c}34,61 \\
-4,3\end{array}$ & $\begin{array}{r}29,79 \\
-12,27\end{array}$ & ${ }^{*} p=0,125^{1}$ \\
\hline Nivel educacional (cuidador; > 8 años, \%) & $\begin{array}{c}78,0 \\
\text { S/D: } 15,0\end{array}$ & $\begin{array}{c}85,0 \\
\text { S/D: } 10\end{array}$ & $\begin{array}{c}79,2 \\
\text { S/D: } 14,2\end{array}$ & ${ }^{*} p=0,001^{2}$ \\
\hline Previsión FONASA (\%) & $\begin{array}{c}38,0 \\
\text { S/D: } 19 \%\end{array}$ & $\begin{array}{c}35,0 \\
\text { S/D: } 10 \%\end{array}$ & $\begin{array}{c}37,5 \\
\text { S/D: } 17,5 \%\end{array}$ & ${ }^{*} p=0,762^{2}$ \\
\hline
\end{tabular}

*Diferencia de promedios entre muestra general y muestra clínica es estadísticamente significativa. S/D: Sin dato. 1. Prueba U de MannWhitney. 2. Chi-cuadrado.

Tabla 2. Puntajes promedio de pruebas estudiadas en muestra general y clínica

\begin{tabular}{|c|c|c|c|c|}
\hline \multirow[t]{2}{*}{ Prueba } & Muestra general & Muestra clínica & Total & \multirow[t]{2}{*}{ Valor $\mathrm{p}^{1}$} \\
\hline & $n=100$ & $n=20$ & $n=120$ & \\
\hline \multicolumn{5}{|c|}{ M-CHAT-R/F: } \\
\hline $\begin{array}{ll}\text { - } & \text { Media } \\
\text { - } & \text { DE }\end{array}$ & $\begin{array}{c}0,81 \\
-1,21\end{array}$ & $\begin{array}{l}9,10 \\
-3,12\end{array}$ & $\begin{array}{r}2,19 \\
-3,52\end{array}$ & 0,000 \\
\hline \multicolumn{5}{|l|}{ ADOS-2: } \\
\hline $\begin{array}{ll}\text { - } & \text { Media } \\
\text { - } & D E\end{array}$ & $\begin{array}{l}2,70 \\
3,36\end{array}$ & $\begin{array}{c}15,00 \\
5,81\end{array}$ & $\begin{array}{l}8,42 \\
7,73\end{array}$ & 0,000 \\
\hline
\end{tabular}

1. Prueba U de Mann-Whitney 


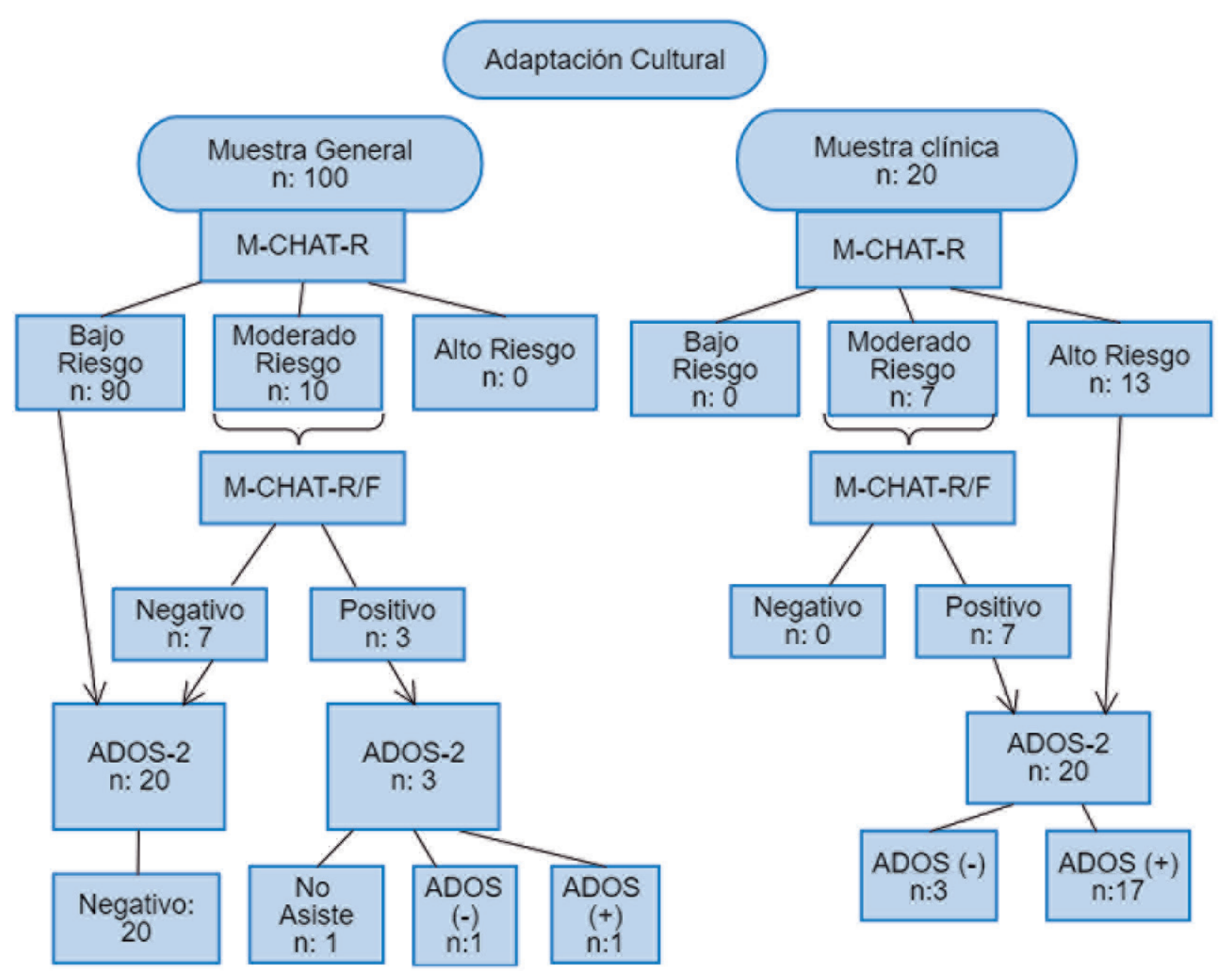

Figura 1. Flujograma de procedimiento. Flujograma que indica resultados del tamizaje en muestra general y clínica $(n=120)$. A ambas muestras se aplicó M-CHAT-R; en aquellos con puntaje de moderado riesgo (puntajes $\geq 3$ y $<8$ ) se realizó entrevista de seguimiento R/F. Luego se realizó test ADOS-2 en todos aquellos que resultaron positivos en M-CHAT-R/F y a 20 niños de la muestra general reclutados aleatoriamente. En la muestra general hubo un resultado ADOS-2 negativo y un ADOS-2 positivo, un caso no completó la evaluación. En la muestra clínica 3 pacientes resultaron negativos y 17 positivos para TEA, según ADOS-2.

descartando el diagnóstico en otro caso. El tercer caso abandonó el protocolo previo a evaluación diagnóstica.

Para detectar falsos negativos, se seleccionaron 20 casos al azar de la muestra de la población general a los que se les aplicó el test ADOS-2, todos con resultados en la categoría Poco o Ningún Riesgo de TEA.

En la muestra de sospecha clínica de TEA, tanto el M-CHAT-R como R/F fueron positivos en todos. A esta muestra se le realizó evaluación clínica y test de ADOS-2. En 3 casos, el resultado fue Poco o Ningún riesgo de TEA y de Alto riesgo de TEA en los 17 restantes. En estos tres casos falsos positivos, se observó que eran pacientes con retraso en el desarrollo del lenguaje, o sea otro trastorno del neurodesarrollo, pero que no interfiere en la comunicación pragmática ni en la reciprocidad social.

\section{Confiabilidad}

Se analizó la consistencia interna para todos los ítems del M-CHAT-R y para el R/F con un resultado considerado como adecuado, (Alfa de Cronbach $=$ $0,889)$. Este resultado se encuentra por sobre el nivel de fiabilidad mostrado por el instrumento original en esta misma versión $(\text { Alfa }=0,79)^{14}$.

\section{Validez}

Validez discriminante: El contraste de M-CHATR/F con la sospecha clínica de TEA, mostró una validez discriminante de $100 \%$ (Tabla 3 ). 
Validez concurrente: M-CHAT-R/F con la prueba diagnóstica ADOS-2 muestran una alta correlación entre sus puntajes ( Pearson $r=0,849 ; \mathrm{p}=0,0001$ ) y la sensibilidad y especificidad concurrente fue de $100 \%$ y $83,3 \%$ respectivamente (Tabla 3 ).

\section{Discusión}

En este trabajo continuamos con el proceso de validación del M-CHAT-R/F, ya iniciado con la adaptación cultural al español-Chile ${ }^{25,26}$. Contar con un instrumento de tamizaje que facilite la pesquisa precoz de los TEA podría permitir intervenciones tempranas y mejores pronósticos en el desarrollo de los niños con TEA. El M-CHAT-R/F ha demostrado a nivel global cumplir con este propósito ${ }^{30}$.

En términos generales el instrumento chileno mostró una adecuada consistencia interna, y logra discriminar con éxito a la población sana de aquella con algún trastorno de desarrollo (Tabla 3).

$\mathrm{Al}$ compararlo con un instrumento estandarizado para el diagnóstico de TEA como el ADOS-2, se comportó con niveles de sensibilidad y especificidad elevados y similares al M-CHAT-R/F original creado por Robins et $\mathrm{al}^{14}$.

Vale la pena destacar que en la población clínica, los falsos positivos obtenidos con el M-CHAT-R/F resultaron tener otros tipos de Trastornos del Neurodesarrollo, de manera similar a lo descrito en la validación del instrumento original de Robins et a ${ }^{31}$. Por lo tanto, se trata de niños que de igual manera requieren evaluación e intervenciones por equipos especializados en desarrollo infanti ${ }^{32}$.

La diferencia observada en la proporción hombre:mujer en nuestro estudio excede lo observado en la literatura, que reporta, inclusive en los últimos estudios de prevalencia ${ }^{33}$, la relación descrita previamente en pacientes con TEA de 4 hombres por 1 mujer. Sin embargo, en menores de 30 meses, esta relación no es tan consistente debido a una tendencia observada a la detección más tardía de los casos de TEA en el género femenino ${ }^{34}$. La identificación de los sesgos involucrados en esta diferencia respecto al diagnóstico temprano de TEA entre niños y niñas sustenta el desarrollo de líneas investigativas actuales ${ }^{35}$.

Se observa que el promedio de edad de los cuidadores de los niños de la muestra clínica es considerablemente mayor que la muestra general, este dato es concordante con lo descrito en la literatura, donde la edad de los padres es un factor de riesgo para TEA ${ }^{36}$. Por último, en esta muestra tenemos una población que en su mayoría presenta cuidadores con estudios superiores técnicos o universitarios, algo que no es representativo necesariamente de la realidad chilena ${ }^{37}$,
Tabla 3. Validez discriminante y concurrente de M-CHAT

\begin{tabular}{|c|c|c|c|}
\hline \multirow[t]{2}{*}{ Prueba } & $\begin{array}{c}\text { Muestra } \\
\text { general }\end{array}$ & $\begin{array}{c}\text { Muestra } \\
\text { clínica }\end{array}$ & \multirow[t]{2}{*}{ Total } \\
\hline & $n=100$ & $n=20$ & \\
\hline M-CHAT alterado al seguimiento: $n$ & 3 & 20 & 23 \\
\hline Validez discriminante (\% total) & & 100,0 & \\
\hline \multirow[t]{2}{*}{ Prueba } & ADOS + & ADOS- & Total \\
\hline & $\mathrm{n}$ & $n$ & $n$ \\
\hline M-CHAT alterado al seguimiento & 18 & 4 & 22 \\
\hline M-CHAT no alterado al seguimiento & 0 & 20 & 20 \\
\hline Sensibilidad (\%) & 100,0 & & \\
\hline Especificidad (\%) & 83,3 & & \\
\hline
\end{tabular}

pero que podría caracterizar a la población usuaria de la Red de Salud UC-Christus.

Dentro de las limitaciones del estudio es importante señalar el número reducido de la muestra, acotado principalmente por la disponibilidad de pacientes menores de 30 meses con sospecha clínica de TEA en nuestra red. Con esta dificultad es que diseñamos un estudio con una relación 5:1 entre la muestra general y clínica. En ese sentido se sugiere incorporar otros contextos nacionales para describir el comportamiento del M-CHAT-R/F en una población más amplia y representativa de la realidad chilena. Otro problema metodológico al que nos enfrentamos en el diseño fue la dificultad para hacerlo ciego a la aplicación del ADOS-2, ya que los padres estando en conocimiento del resultado del tamizaje previo, acudían con la duda diagnóstica a esta evaluación, reconociéndose como fuente de sesgo de sobreinterpretar la relación entre ambos instrumentos. Ambas dificultades señaladas son una barrera común en los estudios de validación de instrumentos de tamizaje/diagnóstico de TEA en este rango etario siendo este un desafío metodológico importante a superar ${ }^{29}$.

A pesar de las dificultades anteriormente señaladas, este trabajo ha repercutido en que se inicie una capacitación a profesionales de salud que está desarrollándose en la atención primaria para implementar esta versión adaptada del M-CHAT-R/F en el Programa Control Niño Sano de 18 meses en aquellos que presentan factores de riesgos de TEA: niños o niñas que presentan algún rezago en las áreas lenguaje y/o social en el test Escala de Evaluación del Desarrollo Psicomotor (EEDP), hermanos e hijos de pacientes con TEA, como también en aquellos casos donde haya directamente una sospecha clínica. Por otro lado, el presente estudio, es considerado también un paso inicial, pero importante para la realización de estudios de prevalencia de TEA en Chile en población menor de 30 meses. 
Determinar la prevalencia del TEA, aún desconocida en nuestro país, nos permitirá dimensionar la necesidad y poder distribuir los recursos necesarios tanto desde la implementación de dispositivos de salud especializados, como también de políticas públicas que contribuyan al bienestar de estos pacientes y sus familias.

\section{Conclusión}

El M-CHAT-R/F en su versión chilena resultó fiable, sensible y específico de manera similar a lo descrito en el estudio original, permitiendo así contar en Chile con un instrumento validado de tamizaje para el Trastorno del Espectro Autista. Se observaron ciertas limitaciones metodológicas como la muestra reducida y la dificultad de ser ciego en la evaluación diagnóstica. Aunque la validación de instrumentos clínicos es un proceso continuo que se perfecciona constantemente, este primer paso contribuye con dos aportes muy significativos: a nivel de la práctica clínica, permite mejorar la capacidad diagnóstica en la detección precoz de TEA, y a nivel de salud pública, la aplicación de un mismo instrumento de tamizaje validado logra estandarizar la detección precoz en la atención primaria. De esta manera se puede realizar un diagnóstico temprano, mejorar el pronóstico y por lo tanto la carga de salud.

\section{Responsabilidades éticas}

Protección de personas y animales: Los autores declaran que los procedimientos seguidos se conformaron a las normas éticas del comité de experimentación humana responsable y de acuerdo con la Asociación Médica Mundial y la Declaración de Helsinki.
Confidencialidad de los datos: Los autores declaran que han seguido los protocolos de su centro de trabajo sobre la publicación de datos de pacientes.

Derecho a la privacidad y consentimiento informado: Los autores han obtenido el consentimiento informado de los pacientes y/o sujetos referidos en el artículo. Este documento obra en poder del autor de correspondencia.

\section{Conflicto de intereses}

Los autores declaran no tener conflicto de intereses.

\section{Agradecimientos}

A los departamentos de Psiquiatría, Pediatría y Salud pública de la Pontificia Universidad Católica de Chile por el apoyo en la realización del trabajo.

Al Dr. Manuel Arriaza, Neurólogo Pediátrico del Hospital Sótero del Río y Red Salud UC-Christus por su activa derivación de pacientes para el estudio.

A los cientos de padres y múltiples profesionales que colaboraron en las distintas fases del estudio.

\section{Financiamiento}

Concurso especial de investigación semilla-interdisciplinario 2015, Proyecto PS 08/15 Dra Coelho-Medeiros - Dra Bedregal. Dirección de Investigación, Escuela de Medicina. Pontificia Universidad Católica de Chile. Ente que financió el trabajo no tuvo influencia en ningún aspecto del mismo (diseño, recolección, análisis o interpretación de datos, preparación, revisión o aprobación del manuscrito final).

\section{Referencias}

1. Baio J. Prevalence of autism spectrum disorder among children aged 8 years Autism and Developmental Disabilities Monitoring Network, 11 sites, United States, 2010. MMWR SurveillSumm 2014; 63:1-21.

2. Christensen DL, Baio J, Braun KV, et al. Prevalence and Characteristics of Autism Spectrum Disorder Among Children Aged 8 Years-Autism and Developmental Disabilities Monitoring Network, 11 Sites, United States, 2012. MMWR SurveillSumm 2016; 65 :1-23.

3. John W. Harrington and Korrie Allen. The Clinician's Guide to Autism.
Pediatrics in Review. 2014;35:62.

4. K. Limberg, K Gruber and M.

Noterdaeme. The German version of the Child Behavior Checklist 1.5-5 to identify children with a risk of autism spectrum disorder. Autism. 2017; 21(3):368-374.

5. Falkmer T, Anderson K, Falkmer M, Horlin C. Diagnostic procedures in autism spectrum disorders: a systematic literature review. Eur Child Adolesc Psychiatry. 2013; 22:329-340.

6. McPheeters M. L., Weitlauf A., Vehorn A., et al. Screening for Autism Spectrum Disorder in Young Children: A Systematic Evidence Review for the U.S. Preventive Services Task Force. AHRQ 2016; 1305185-E(129):202.
7. Dawson G, Rogers S, Munson J, Smith M Winter J, Donaldson A and Varley J. Randomized Controlled trial of an intervention for toddlers with autism: The early Start Denver Model. Pediatrics. 2010; 125,1:e17-e23.

8. Reichow B, Barton E, Boyd BA and Hume $\mathrm{K}$. Early intensive behavioral intervention (EIBI) for young children with autism spectrum disorders (ASD). Cochrane Database Syst Rev 2012; 10: CD009260.

9. Rogers SJ, Vismara LA. Evidence-based comprehensive treatments for early autism. J Clin Child Adolesc Psychol. 2008;37:8-38.

10. Johnson CP, Myers SM; American Academy of Pediatrics Council on 
Children with Disabilities. Identification and evaluation of children with autism spectrum disorders. Pediatrics. 2007; 120:1183-215.

11. Valicenti-McDermott $\mathrm{M}$, Hottinger $\mathrm{K}$, Seijo R, and Shulman L. Age at diagnosis of autism spectrum disorders. J Pediatr. 2012; 161:554-6.

12. Dumont-Mathieu T, Fein D. Screening for autism in young children: The Modified Checklist for Autism in Toddlers (M-CHAT) and other measures. Ment Retard Dev Disabil Res Rev. 2005;11:253-62.

13. F. Volkmar, M Siegel, M WoodburySmith and B. King. Practice Parameter for The Assessment and Treatment of Children and Adolescent with Autism Spectrum Disorder. J. Am. Acad. Child Adolesc. Psychiatry. 2014, 53 (2).

14. Robins D, Casagrande K, Barton M, Chen C, Thyde P. Follow-up (M-CHAT-R/F) Validation of the Modified Checklist for Autism in Toddlers. Pediatrics 2014; 133(1) :37-47.

15. Fuentes J, Bakare M, Munir K, Aguayo P, Gaddour N. Autism spectrum disorder. En Rey JM (ed), IACAPAP e-Textbook of Child and Adolescent Mental Health. Geneva: International Association for Child and Adolescent Psychiatry and Allied Professions 2014.C2:1-35.

16. Ministerio de Salud. Guía de Práctica Clínica de Detección y Diagnóstico Oportuno de los trastornos del Espectro Autista (TEA). Santiago, 2011. Disponible en: http://www.minsal.cl/portal/url/item/ bd81e3a09ab6c3cee040010164012ac2.pdf. última visita 10-09-2018.

17. Generalitat de Catalunya. Dirección General de Planificación e Investigación en Salud. Departamento de Salud. Plan de atención a las personas con Trastorno del Espectro Autista. Barcelona, 2013. Disponible en: http://canalsalut.gencat/ web/.content/home_canal_salut/ profesionals/termes_de_salut/salut_ mental/documents/pdf/pla_tea_2012-pdf. última visita 10-09-2018.

18. Haute Autorité de Santé. Méthode Recommandations pour la pratique clinique. Trouble du spectre de l'autismeSignes d'alerte, repérage, diagnostic et évaluation chez l'enfant et l'adolescent. Février 2018. Disponible en: http:// www.has-sante.fr/portail/upload/docs/ application/pdf/21002/tsa_-_des_signes_ dalerte_a_la_consultation_dediee_en_ soins_primaires_ler_ligne_synthese_-pdf. última visita 08-09-2018.

19. Zwaigenbaum L, Bauman ML, Stone W, et al. Early identification of autism spectrum disorders: Recommendations for practice and research. Pediatrics. 2015;136.Suppl 1:S10-40.

20. Cuesta-Gomez JL, Manzone LA, Posada-De-La-Paz M. Modified checklist for autism in toddlers cross-cultural adaptation for Argentina. International journal of Developmental Disabilities. 2016;62(2):117-123.

21. Albores-Gallo L, Roldán-Ceballos O, Villareal-Valdes G, et al. M-CHAT Mexican version validity and reliability and some cultural considerations. ISRN Neurol. 2012;2012: ID 408694.7.

22. Backes B, Gomes Mônego B, Alves Bosa C, et al. Psychometric properties of assessment instruments for autism spectrum disorder: a systematic review of Brazilian Studies. J Bras Psiquiatr. 2014;63(2):154-64.

23. Canal-Bedia R, García-Primo P, MartinCilleros MV, et al. Modified checklist for autism in toddlers: cross-cultural adaptation and validation in Spain. J Autism Dev Disord. 2011;41(10):13421351.

24. Ministerio de Salud de Chile. Norma Técnica para la supervisión de niños y niñas de 0 a 9 años en la Atención Primaria de Salud Programa Nacional de Salud de la Infancia. № 1 66, Resolución Exenta No 336, Santiago, 2014

25. Coelho-Medeiros E, Bronstein J, Aedo K et al. Relevancia de la adaptación cultural en la validación del M-CHAT-R/F como instrumento de tamizaje para autismo. Rev Chil Pediatri. 2017;88(6):822-33.

26. Robins D, Frein D, Barton M. M-CHAT$\mathrm{R} / \mathrm{F}$ (Internet). Adaptación por el Equipo TEA UC. (2017). M-CHAT-R/F. Español-Chile. Disponible en: http:// www.mchatscreen.com/wp-content/ uploads/2017/01/M-CHAT-R_F_EspanolChile2017.pdf. última visita 10-09-2018.

27. Asociación Americana de Psiquiatría. Guía de consulta de los criterios diagnósticos del DSM 5. Arlington, VA, Asociación Americana de Psiquiatría, 2013.

28. Lord C, Rutter M, DiLavore PC, et al.
ADOS-2. Escala de Observación para el Diagnóstico del Autismo - 2. Manual (T. Luque, adaptadora). Madrid: TEA Ediciones. 2015.

29. Randall M, Egberts KJ, Samtani A, et al. Diagnostic tests for autism spectrum disorder (ASD) in preschool children. Cochrane Database of Systematic Reviews 2018, Issue 7. Art. No.: CD009044.DOI: 10.1002/14651858.CD009044.pub2.

30. Zwaigenbaum L, Bauman ML, Fein D, et al. Screening of Autism Spectrum Disorder: Recommendations for Practice and Research. Pediatrics. 2015; (supl 1):S41-S59.

31. Robins D. Screening for autism spectrum disorders in primary care settings. Autism. 2008; 12(5):537-56.

32. Courtenay Frazier Norbury and Rhea Paul. Disorder of Speech, language, and communication. En: Anita apar and Daniel S. Pine, James F. Leckman, Stephen Scott, Margaret J. Snowling, Eric Taylor. Rutter's Child and Adolescent Psychiatry, 6th Edition. Editorial WileyBlackwemm. 2015. C52:683-701.

33. Baio J, Wiggins L, Christensen DL. et al. Prevalence of Autism Spectrum Disorder Among Children Aged 8 YearsAutism and Developmental Disabilities Monitoring Network, 11 Sites, United States, 2014. MMWR Surveill Summ. 2018;67(6):1-23. Published online 2018 Apr 27. doi: 10.15585/mmwr.ss6706a.

34. Dworzynski K, Ronald A, Bolton P, Happé F. How Different Are Girls and Boys Above and Below the Diagnostic Threshold for Autism Spectrum Disorders?. J. Am. Acad. Child Adolesc. Psychiatry. 2012;51(8):788-97.

35. Fultona A, Paynterb J, Trembathd D. Gender comparisons in children with ASD entering early intervention. Research in Developmental Disabilities. 2017;68:2734.

36. Guinchat V, Thorsen P, Laurent C, Cans C, Bodeau N, Cohen D. Pre-, Peri- and neonatal risk factors for autism. Acta Obstet Gynecol Scand. 2012; 91:287-300.

37. Ministerio de Educación (MINEDUC). Análisis de indicadores educativos de Chile y la OCDE en el contexto de la reforma educacional. Serie Evidencias N. 31, 2015. Recuperado en: https/centro estudios.mineduc.cl/publicacones-ce/ evidencias. Última visita 10-10-2018. 